\title{
LA PRESUPUESTACIÓN POR PROGRAMAS EN LA COMUNIDAD AUTÓNOMA DE LA RIOJA ${ }^{1}$
}

\author{
$\mathrm{M}^{\mathrm{a}}$ DOLORES PIÑA GARRIDO \\ ProfesoraTitular de Derecho Financiero y Tributario \\ Universidad de La Rioja
}

SUMARIO

1. INTRODUCCIÓN, 2. LA TÉCNICA DE LA PRESUPUESTACIÓN POR PROGRAMAS EN LA COMUNIDAD AUTONÓMA DE LA RIOJA: TEORÍA Y PRÁCTICA. 3. EFECTOS DE LA APROBACIÓN DE LA NUEVA LEY GENERAL PRESUPUESTARIA DEL ESTADO EN LA RIOJA, 4. CONCLUSIONES: ¿ES DESEABLE AVANZAR HACIA UNA GESTIÓN POR OBJETIVOS?.

\section{INTRODUCCIÓN}

En la Comunidad Autonóma de La Rioja no se ha promulgado, a diferencia de lo que ha sucedido en otras CCAA, una Ley de Hacienda Pública en la que se regulen los aspectos básicos de la gestión de los recursos públicos, presupuestación y control incluidos, siguiendo el modelo de la Ley General Presupuestaria del Estado y haciendo uso de la competencia que el T IV de su Estatuto de Autonomía -LO 3/1982, de 9junio- le reconoce. De modo que es la LGP del Estado la que se aplica como Derecho supletorio, excepto en aquellas cuestiones que la Comunidad decide regular de modo específico, normalmente en las leyes anuales de presupuestos como hace por ejemplo con las modificaciones presupuestarias.

\footnotetext{
${ }^{1}$ Trabajo cerrado en enero de 2004.
} 
Esto significa que la forma, plazos y procedimiento de elaboración de los presupuestos de la CA son los regulados en la LGP del Estado con el complemento de las órdenes y resoluciones emanadas de la Consejería de Hacienda, que únicamente pueden actuar dentro de los márgenes que la ley -estatal- permite. Dada la vigencia del principio de reserva de ley en este campo -art. 133 y $134 \mathrm{CE}$, entre otros, y 56 del Estatuto de Autonomía de La Rioja-, si la CA quiere establecer una regulación distinta a la del Estado, debe hacerlo mediante la aprobación de una ley propia, no a través de las órdenes anuales de la Consejería de Hacienda con las instrucciones para la elaboración de los presupuestos del ejercicio siguiente $\mathrm{y}$, menos aún, mediante resoluciones de las diferentes Direcciones Generales de esta Consejería. Y si nos detenemos en comentar este punto es porque, como tendremos ocasión de ver y como, por otro lado, ha sucedido también hasta ahora en el Estado, la regulación de los procedimientos de elaboración, gestión y control de los presupuestos se ha llevado a cabo a través de normas del más bajo rango y de simples resoluciones administrativas, sin tener en cuenta no ya la vigencia del principio de reserva de ley presupuestaria que acabamos de citar, sino la incidencia en este área del art. 31.2 CE.

La inexistencia de una ley de gasto público propia de la CA significa también que los cambios que se introducen en la normativa estatal afectan inmediatamente a La Rioja y esta la razón por la que hemos decidido escribir este trabajo. Recientemente se ha aprobado una nueva Ley General Presupuestaria, L 47/2003, 26-noviembre, que sustituye al hasta ahora vigente Texto Refundido de LGP, RDLeg 1091/1988, de 23 de septiembre. Muchos de los preceptos que vamos a comentar en este trabajo han entrado en vigor el 1 de enero de 2004, varios sólo a los efectos de preparar los presupuestos del 2005 -vid DF $5^{\mathrm{a}}$-.

Por tanto, y mientras la CA no disponga algo distinto -mediante la aprobación de una Ley de Hacienda Pública propia o utilizando cualquier otro tipo de ley, por ejemplo, la Ley de los Presupuestos Generales de 2005-, la reforma en materia de presupuestación, gestión y control del gasto público que se está llevando a cabo a nivel estatal le obligará a cambiar, también ya para los presupuestos de 2005, sus formas de gestión. Y las innovaciones son de cierta relevancia, puesto que lo que el Estado pretende es sustituir el sistema de presupuestación por programas por un modelo de gestión por objetivos.

\section{LA TÉCNICA DE LA PRESUPUESTACIÓN POR PROGRAMAS EN LA COMUNIDAD AUTONÓMICA DE LA RIOJA: TEORÍA Y PRÁCTICA.}

Las leyes que aprueban los presupuestos públicos representan, pese a todas sus limitaciones, una de las grandes conquistas del Estado democrático de Derecho, puesto que impiden que los gobiernos puedan poner en marcha ningún tipo de iniciativa que soponga un coste económico -cualquiera, pues- sin haber sido antes aprobada por el Parlamento. Es cierto que las facultades del Parlamento se encuentran bastante cercenadas - no tiene derecho de iniciativa legislativa, no puede presentar ciertas enmiendas sin cumplir determinados requisitos, etc, como imponen los art. $134 \mathrm{CE}$ y 56 del Estatuto de Autonomía de La Rioja-, que en una democracia de partidos la mayoría 
parlamentaria no está demasiado interesada en crear problemas a "su" gobierno, y que la ley aprobada en enero puede experimentar y de hecho experimenta numerosas modificaciones a lo largo del año, pero aún así, la ley de presupuestos es aquella que recoge todos los ingresos que se prevén recaudar durante el año siguiente y todos los gastos que se autoriza a realizar, de modo que muy bien puede afirmarse sin exagerar que lo que no existe en la ley de presupuestos no existe en el mundo y que, por tanto, el momento de la discusión y aprobación del proyecto de presupuestos presentado por el Gobierno cada año es uno de los más importantes del ejercicio parlamentario.

Para que el Parlamento pueda en verdad desarrollar su papel es preciso que el Gobierno sea leal y le entregue toda la información útil de que dispone sobre el proyecto de presupuestos que presenta y que el Parlamento pueda y quiera conocer y asimilar dicha información necesaria para la toma de decisiones razonables y razonadas.

Y para esto, al margen de otros factores, la forma en que se preparan los presupuestos -qué información se incluya, de qué manera se ordenen los ingresos y gastos públicos, qué documentación complementaria se aporte, cómo se estructura el proyecto de presupuestos, en definitiva- resulta esencial.

También el desarrollo de las tareas de gestión del presupuesto -el empleo de recursos públicos en la cobertura de fines asimismo de interés público- se verá influido por la forma de preparación de los presupuestos, esto es, la propia Administración podrá desarrollar su labor con mayor economía, eficacia y eficiencia o no según la técnica presupuestaria que se haya empleado en la elaboración de los presupuestos.

Tampoco el control, tercera y última fase del ciclo presupuestario, queda al margen de la técnica presupuestaria empleada, de modo que la información que puede proporcionar al Gobierno y, en última instancia, al Parlamento para la toma de decisiones sobre el presupuesto de años sucesivos no será la misma según el tipo de presupuesto utilizado.

Existen muy variadas técnicas presupuestarias que van desde el presupuesto de medios tradicional, , hasta la más moderna gestión por objetivos, pasando, básicamente, por el presupuesto por tareas y el presupuesto por programas.Todas ellas han sido utilizadas en nuestro país.

2.A. La presupuestacion por programas en la comunidad autonoma de la rioja: en teoria.

En la Comunidad Autónoma de La Rioja se viene intentando utilizar desde 1992 el presupuesto por programas -en el Estado se comenzó a utilizar en 1984-, pero con un éxito sólo formal, como veremos. Antes quisiéramos destacar que la elección de técnica presupuestaria debe estar guiada por los principios que, según el art. $31 \mathrm{CE}$, deben respetar los poderes públicos en todas las fases del ciclo presupuestario -aprobación, gestión y control del gasto público- $\mathrm{y}$ en todos los niveles de gobierno, también en el autonómico, a saber, economía -esto es, ahorro de recursos, no despilfarro-, eficacia -consecución de los objetivos perseguidos- y eficiencia -suma de los dos anteriores, 
obtención de la mayor productividad posible de cada recurso empleado en la consecución de los fines previstos- ${ }^{2}$.

Esto obliga a descartar totalmente el presupuesto de medios, en el que simplemente se toma como referencia el del año anterior y se incrementan todas las partidas en el porcentaje que se estima razonable, lo que le convierte en un presupuesto incrementalista que no satisface ninguno de los valores mencionados -economía, eficacia y eficiencia- y que, además, es insostenible en momentos de crisis económica o en aquellos en los que se busca controlar el déficit público, como sucede ahora por imperativo de Derecho Comunitario ${ }^{3}$.

Pero pueden utilizarse otras técnicas como las que reseñamos brevemente a continuación $^{4}$ : el presupuesto por tareas -Performance Budget- y el presupuesto por programas -Planning, Programming Budget System-.

En el primero el órgano administrativo debe indicar cuáles son las funciones que le competen y las actividades que desarrolla a lo largo del ejercicio presupuestario para las que solicita recursos. Con ello se introduce una cierta dosis de racionalización en la gestión pública, desde el momento en que se obliga a los centros gestores a parar y pensar sobre lo que están haciendo.

En el segundo se exige de los centros gestores que fijen objetivos para su actuación durante el siguiente ejercicio presupuestario y reordenen sus actividades en función de los mismos, en forma de programas. Esto es, se les pide que planifiquen su actuación, a medio plazo, para 3 o 4 años; que detecten y definan las situaciones de necesidad -los problemas económico-sociales- que quieren resolver para aumentar el bienestar y calidad de vida de los ciudadanos a cuyo servicio están; que se fijen una meta, que deben descomponer en objetivos estratégicos y operativos; que definan sus planes de acción para conseguir alcanzar esos objetivos, y que incluyan indicadores, elementos que permitirán conocer qué resultados están consiguiendo y así comprobar en qué medida están alcanzando sus objetivos. Y todo ello para poder decidir qué se hace con el programa en años sucesivos: si se mantiene, se modifica o se suprime y por qué alternativa.

Esta es la técnica que, como adelantábamos, se ha utilizado en los últimos años en nuestro país, a nivel estatal y autonómico -no local-, al menos en teoría. No es una técnica perfecta, porque puede dar lugar a rigideces, puede impedir que los órganos administrativos se adapten con la suficiente rapidez a situaciones cambiantes, obligándoles a mantener con vida programas que no están dando resultado, ni pueden darlo. Por esto se defiende en algunos ámbitos la sustitución del PPBS por la gestión por objetivos -Management by Objectives-, técnica que se introduce en la nueva Ley General Presupuestaria -se pretende su empleo ya en la preparación de los presupuestos

\footnotetext{
${ }^{2}$ Hemos tomado las definiciones de SUBIRATS, "Análisis de políticas públicas y eficacia de la Administración", INAP, 1994 y de otros autores que citamos ampliamente en nuestro libro "La presupuestación por programas y el control interno de economía, eficacia y eficiencia en España", Tecnos/Universidad Pública de Navarra, 1998.

${ }^{3}$ Vid art 104 Tratado de la Comunidad Europea y el Pacto de Estabilidad y Crecimiento contenido en la resolución del Consejo Europeo de Amsterdam de 7 de junio de 1997 y los reglamentos CE del Consejo 1466/1997 y 1467/1997, de 7 de julio.

4 Para más información véase nuestro libro "La presupuestación..." ya cit y la bibliografía allí mencionada.
} 
de $2005^{5}$ - y en la que los recursos públicos se ponen a disposición del órgano administrativo para que este pueda conseguir unos objetivos que son los únicos que aparecen determinados en la ley de presupuestos. Las actuaciones que sean necesarias para llegar a ellos se dejan a la libre disposición y decisión del ente que, a cambio, se responsabiliza de la consecución de los resultados previstos. En caso de no alcanzarlos pierde recursos en los presupuestos de los ejercicios siguientes -vid exposición de motivos y los art. 26-41 de la nueva LGP-.

En la Rioja se comenzaron a utilizar los presupuestos por programas en 1992, con carácter vinculante y para el conjunto de su Administración -nosotros vamos a centrarnos en el núcleo duro, dejando al margen organismos autónomos y otro tipo de entes públicos-, después de un curso de preparación a funcionarios seleccionados de las distintas Consejerías, donde se les explicó en qué consistía la nueva técnica presupuestaria, se les informó acerca del procedimiento de elaboración, se les proporcionaron las fichas de programas -documentos en los que se condensa la información esencial sobre la estructura de un programa presupuestario-, y se les ofrecieron ejemplos prácticos relacionados con distintas áreas de actuación ${ }^{6}$.

El Estado unos años antes -desde 1984- había preferido una introducción progresiva, primero sin efectos vinculantes - hasta 1989-, comenzando por Ministerios en los que era de esperar que el proceso de adaptación a la nueva técnica no fuera demasiado complicado por tratarse de órganos administrativos cuya actuación produce bienes y servicios públicos tangibles y directamente perceptibles por los ciudadanos -Obras Públicas, Educación, Sanidad-, y respecto a la cual podían aplicarse categorías como objetivos, resultados o indicadores sin demasiados problemas.

Que La Rioja optara por aplicar de un año para otro un sistema de presupuestación tan complejo como el PPBS, con carácter vinculante además, podría pensarse que se debía al hecho de que, al fin y al cabo sólo se trataba de extender a la Comunidad algo ya sobradamente conocido y aplicado a nivel estatal. Lamentablemente esto no era así. Esta técnica presupuestaria es muy compleja, tanto que ni siquiera hoy en día -veinte años después de su introducción- en el Estado todos los centros gestores la aplican correctamente -mucho nos tememos que sólo lo hace en realidad una minoría ${ }^{7}$ - y esto no sucede sólo en España, sino en muchos otros países, como demuestran los estudios de la $\mathrm{OCDE}^{8}$.

Y es que, como toda reforma administrativa, la implantación de una nueva técnica presupuestaria $-\mathrm{y}$ es importante no olvidar esto precisamente en estos momentosrequiere todo un cambio de cultura y modos de hacer en el seno de una institución muy

\footnotetext{
${ }^{5}$ Vid nuestros comentarios al respecto en "Hacia una gestión por objetivos con la nueva Ley General Presupuestaria" (2004).

${ }^{6}$ Los ejemplos proporcionados se referían a programas como Gabinete del Presidente, Vicepresidencia del Gobierno, Administración General de Hacienda y Economía, Presupuestación, Conservación y Explotación de Carreteras, Coordinación y Promoción del Turismo, Promoción y Cooperación Cultural, Dirección y Organización de la Función Pública, Organización de la Estructura y Sistemas de Producción e Industrialización Agraria y Ganadera, y Ordenación, Control e Información del Consumidor.

${ }^{7}$ Vid nuestro artículo "Hacia una gestión por objetivos con la nueva Ley General Presupuestaria", 2004.

8 Vid pe OECD, "Overview of Results-focused Management and Budgeting in OECD Member Countries", 2002; PERRIN, B, "Implementing the Vision: Addressing Challenges to Results-focused Management and Budgeting", 2002; ZAPICO GOÑI, "Budget for Results in Spain: Lessons learned after two Decades of Reform", OECD, 2003.
} 
compleja como es la Administración Pública -también la autonómica, aunque sus dimensiones sean mucho más reducidas- y ese cambio de cultura exige preparación, asistencia técnica y formación de los funcionarios implicados -de todos, no sólo de los que preparan los presupuestos del ejercicio siguiente, en las oficinas presupuestarias, puesto que las novedades afectan a todo el proceso de gestión y también de control del gasto público-, apoyo constante, liderazgo y ofrecimiento de un modelo en el propio comportamiento del órgano que centralice o lleve la iniciativa del cambio -en materia presupuestaria, la Consejería de Hacienda-. Y requiere una evaluación del proceso de reforma mientras este se está llevando a cabo, para comprobar los efectos que se están produciendo, las desviaciones y sus causas, para pensar en alternativas...

Ese proceso de evaluación ha faltado tanto en el Estado, como en la Comunidad. Pero es que en esta además han faltado la preparación previa y la formación continuada de todos los centros gestores, que sólo han contado como apoyo cada año con las órdenes dictadas por la Consejería de Hacienda con las instrucciones para la elaboración de los Presupuestos Generales de la Comunidad para el año siguiente, y las resoluciones de la Dirección General de Presupuestos.

En esas órdenes anuales ${ }^{9}$ se menciona expresamente la técnica del Presupuesto por Programas ${ }^{10}$ como aquella que deben emplear los centros gestores en la elaboración de sus anteproyectos de presupuestos, y se ordena definir y concretar objetivos y actividades, así como establecer indicadores para el seguimiento del grado de realización de los primeros ${ }^{11}$, y rellenar las fichas oportunas ${ }^{12}$. Pero no se dan mayores detalles sobre las características que deben reunir esos elementos, que son los que conforman un programa presupuestario. Por otro lado, la supuesta clasificación funcional y por programas que aparece en el anexo I no incluye un solo programa ${ }^{13}$.

Y ha sido al menos desde $1993^{14}$. Parece que no se ha producido evolución alguna en el diseño y aplicación de la técnica de PPBS en la Comunidad desde entonces -las órdenes anuales de la Consejería de Hacienda son idénticas año tras año-, lo cual no deja de ser extraño, puesto que lo lógico -y así ha sucedido a nivel estatal- hubiera sido que los inevitables errores de los primeros años hubieran dado lugar a la introducción de mejoras o cambios de dirección en la preparación de los presupuestos de los años siguientes.

\footnotetext{
${ }^{9}$ Vid pe la de 14 de mayo de 2003, por la que se dictan las normas para la elaboración de los Presupuestos Generales de la Comunidad Autónoma de La Rioja para 2004.

${ }^{10}$ Vid punto 1 de la Orden para los presupuestos de 2004.

${ }^{11}$ Punto 1.2 , letras a y b de la Orden para los presupuestos de 2004.

12 Punto 2.1 de la Orden para los presupuestos de 2004, que, por cierto, se remite a unos anexos que no se publican en el BOR -la nota final incluída explica literalmente: "No se acompañan las informaciones recogidas en los anexos II y III por conocerse en los ámbitos administrativos en que se utilizan”, lo cual no deja de ser sorprendente.

${ }^{13}$ En efecto, lo que aparece allí es solamente la clasificación funcional -aunque se anuncie en el título del anexo que es también por programas- y recoge grupos de funciones -pe grupo 4: producción de bienes públicos de carácter social-, funciones -pe función 4.3: vivienda y urbanismo-, y subfunciones -pe 4.3.1 vivienda-.

${ }^{14}$ Desgraciadamente no hemos podido localizar la Orden para los presupuestos de 1992, que fue la primera en regular la presupuestación por programas en La Rioja.
} 
Hubo un momento en se intentó dar un paso adelante, que, en nuestra opinión, hubiera sido muy positivo. En 1997 se elaboró una "Guía orientativa para los presupuestos de 1998" - presentada por el entonces Consejero de Hacienda y Promoción Económica, Félix Revuelta Segura-, en la que se quiso enlazar el programa político del partido que había ganado las elecciones, con los programas de actuación sectorial de las distintas Consejerías y el presupuesto por programas. Se pensó que era necesario enmarcar los presupuestos anuales en el seno de una planificación política y económica plurianual que debía comenzar con un "Plan Estratégico de La Rioja", con el que ya se contaba y en cuya elaboración habían participado incluso los agentes sociales y económicos de la Región, con estrategias para períodos de 10 o 15 años. En ese plan se efectuaba un diagnóstico estratégico de la $\mathrm{CA}$-sus puntos críticos positivos y negativos desde el punto de vista económico, social, productivo, geográfico...- y se formulaban estrategias de desarrollo. Pues bien, la idea era (1) partir de las líneas principales de estas estrategias de desarrollo del Plan -pe línea 1.6: apoyar el desarrollo de las infraestructuras que mejoren la competitividad-, (2) para elaborar planes o programas de actuación -pe 1.6.3 Plan de autogeneración energética, 1.6.4. Programa de apoyo a la creación de infraestructuras turísticas-, (3) que enlazaran con el programa del nuevo partido político en el poder - programa que pe contenía ideas como la de potenciar las políticas orientadas a un mejor aprovechamiento energético, o aumentar la capacidad hotelera existente en La Rioja y abrir oficinas turísticas en los principales centros turísticos de la Comunidad-, (4) para fijar objetivos más concretos a partir de ahí -pe disminuir la desventaja de depender energéticamente de otras regiones, aumentando la generación de energía de La Rioja o aprovechar el potencial del sector turístico creando las infraestructuras necesarias para favorecer su desarrollo-, (5) articular en torno a ellos PROGRAMAS DE GASTO -los referentes a nuestros ejemplos eran "Regulación, protección de la propiedad y calidad industrial" y "Coordinación y promoción del Turismo y Comercio"-, (6) ordenar las actividades en ellos -pe estudio de planificación de las fuentes de energía existentes en La Rioja o estudio de la demanda actual y potencial de turismo, planificación de las obras de infraestructura claves, y concurso y adjudicación de las mismas- y determinar con toda claridad quiénes serían los órganos gestores responsables de cada programa.

De este modo se estaban llevando a cabo los esfuerzos de reflexión que un buen sistema de planificación y presupuestación por programas exige, analizando la situación de necesidad sobre la que se quiere actuar y estableciendo las metas, objetivos estratégicos y objetivos operativos en torno a los cuales ordenar las actividades del ente y configurar un programa. El paso siguiente es elegir los indicadores que permitirán comprobar la eficacia, economía y eficiencia del programa.

Sin embargo, esta idea que comentamos se quedó ahí olvidada y no llegó a aplicarse ni a los presupuestos de 1998, ni a ningún otro.

Es posible que los cambios y mejoras que se echan de menos en las normas -en las órdenes de la Consejería de Hacienda anuales con las instrucciones para la elaboración de los presupuestos del ejercicio siguiente en el conjunto de la Administración autónomica- se hayan introducido utilizando vías informales, accesibles en el ámbito de una Comunidad de las dimensiones de la nuestra, al margen de toda regulación normativa. Si esto ha sido así, hemos de decir que no nos parece una forma de actuación loable, dado que la técnica empleada en la elaboración de los presupuestos no es una 
mera cuestión procedimental administrativa con efectos puramente internos, sino que implica una forma de gestión de los recursos públicos en sentido amplio que afecta a todas las fases del ciclo presupuestario, y que debe responder a los principios del art. 31.2 CE, a saber, economía, eficacia y eficiencia, como criterios formales, pero también asignación equitativa de los recursos públicos, principio de justicia material en el gasto que exige que los fines atendidos con el dinero que pertenece a los ciudadanos sean aquellos que cuenten con apoyo y legitimación constitucional -fundamentalmente entre los principios rectores de la política social y económica, art.39 y ss CE-. Y por esto las técnicas de presupuestación y gestión del gasto público utilizadas por la Administración no son indiferentes para los ciudadanos.

2.B. La presupuestacion por programas en la comunidad autonoma de la rioja: en la practica.

Desde el punto de vista de los principios que acabamos de citar del art. $31 \mathrm{CE}$ la técnica de la presupuestación por programas o PPBS es adecuada porque exige a la Administración planificar sus actuaciones una vez identificada una situación de necesidad que requiere intervención pública -y la población destinataria de la misma-, fijar un objetivo -empezar por una meta, y concretar en forma de objetivos estratégicos y operativos-, agrupar actividades en función del o los objetivos, y seleccionar los indicadores - de proceso, resultados e impacto- que permitirán saber en cada momento, al gestor y al responsable político, si el programa público al cual se han destinado determinados recursos está siendo útil a la sociedad o no -útil porque esté resolviendo la situación de necesidad de la que se partía, esto es, útil porque produce un "impacto" social y económico que cambia la realidad; no basta la simple producción de "resultados"-.

¿Responden a estas características los programas de los presupuestos de nuestra Comunidad? Nosotros hemos estudiado los programas de los presupuestos de $2003 \mathrm{y}$ 2004 o lo hemos intentado, puesto que en realidad sólo hemos podido analizar los proyectos de programas. Y que los programas definitivos, los aprobados por el Parlamento, no se publican y sólo son conocidos por los centros gestores responsables. Ni siquiera la Consejería de Hacienda dispone de una copia de la versión definitiva de todos los programs presupuestarios. Si hemos podido acceder al material ha sido gracias a la cortesía de los funcionarios de la Sección de Presupuestos de la DG de Economía y Presupuestos de la Consejería de Hacienda, ya que no se publican, ni editan de ninguna manera. Al igual que sucede en el Estado, la Ley de Presupuestos de la Comunidad no incluye la descripción de los programas. Sólo en un anexo se listan los programas, sin explicar su contenido. Con lo cual surge la pregunta acerca de cuál su eficacia jurídica, dado que, aunque formen parte en teoría de la ley de presupuestos, no se publican y en nuestro ordenamiento jurídico las normas sólo obligan a partir de su publicación completa en los Boletines Oficiales ${ }^{15}$.

\footnotetext{
${ }^{15}$ Vid para más detalles nuestro trabajo "Hacia una gestión por objetivos...".
} 
Hemos seleccionado los programas que nos han parecido más interesantes por sus aspectos positivos o negativos, representativos estos últimos de errores bastante extendidos en el conjunto de los mismos ${ }^{16}$. Y hemos observado lo siguiente:

Muchos de los "programas presupuestarios" no lo son ni siquiera desde el punto de vista formal, pues no respetan la estructura típica de un programa, ni distinguen entre objetivos, actividades e indicadores, como se indica en las órdenes con las instrucciones para la elaboración de los presupuestos del ejercicio siguiente dictadas cada año por la Consejería de Hacienda ${ }^{17}$, y, en ocasiones, hasta se incluyen varios unidos ${ }^{18}$.

Prácticamente ningún programa comienza planteándose una situación de necesidad, un problema socio-económico que requiera la intervención pública y que justifique su existencia. Muchas veces se deja en blanco incluso el apartado que según el modelo impuesto por la Consejería de Hacienda en sus órdenes anuales todos los programas deben contener, dedicado a la descripción general del programa y la

16 - De la Consejería de Obras Públicas, Transportes, Urbanismo y Vivienda (en los presupuestos de 2004 esta Consejería ha sido reformada y la mayor parte de los programas que citamos a continuación se incardinan en la Consejería de Vivienda, Obras Públicas y Transportes):

Programa 5124: Gestión e infraestructura de parque móvil.

Programa 5121: Creación de infraestructuras de carreteras.

Programa 5131: Gestión e infraestructura hidráulica.

Programa 5132: Defensa y tratamiento de riberas.

Programa 4311: Promoción y ayuda para la construcción, rehabilitación y acceso a la vivienda.

Programa 4321: Planeamiento, control y gestión urbana (ahora dependiente de la Consejería de

Programa 4321: Planeamiento, con
-

- De la Consejería de Sanidad y Servicios Sociales (Consejería de Salud en los presupuestos de 2004):

- $\quad$ Programa 4131: Promoción y Protección de la salud.

- $\quad$ Programa 4111: Administración General de salud.

- De la Consejería de Hacienda (Consejería de Hacienda y Empleo en los presupuestos de 2004):

- $\quad$ Programa 6141/38: Administración, gestión tributaria y financiación.

- $\quad$ Programa 61 31: Control interno y contabilidad.

- De la Consejería de Turismo y Medio Ambiente (Consejería de Turismo, Medio Ambiente y Política Territorial en los presupuestos de 2004)

Programa 4432: Medidas preventivas y actuaciones para la protección del medio ambiente.

- De la Consejería de Educación, Cultura, Juventud y Deportes (Consejería de Educación, Cultura y Deportes en los presupuestos de 2004, encargada del programa que nos interesa):

Programa 4223: Enseñanza Universitaria.

${ }^{17}$ Así sucede con los de la Consejería de Educación, que se limita a indicar quiénes son los centros gestores responsables de cada programa y una lista de funciones y actividades mezcladas en cada uno. Pe del programa de Enseñanza universitaria sólo se nos dice que pretende:

“a) Gestionar la Residencia Universitaria "Santo Domingo de la Calzada”, en Logroño.

b) Gestionar la subvención a la universidad de La Rioja, para atender sus gastos corrientes y de capital.

c) Financiar los gastos del Consejo Social de la Universidad de La Rioja.

d) Dotar las becas para estudios universitarios.

e) Colaborar en los gastos de funcionamiento del Centro Asociado de la UNED de La Rioja.

f) Subvencionar a las Escuelas Universitarias de Relaciones Laborales y de Trabajo Social como Escuelas Universitarias adscritas".

${ }^{18}$ Los programas 6141/38, Administración, Gestión Tributaria y Financiera, de la Consejería de Hacienda son un clarísimo exponente de lo que estamos diciendo, pues allí se recogen todas las actividades que desarrolla la Dirección General de Tributos correspondientes al Servicio de Gestión de Tributos Cedidos, Servicio de Tributos Propios y Locales, Servicio de Coordinación de Ingresos y Recaudación y Servicio de Inspección Tributaria y Valoración, siguiendo en realidad la clasificación orgánica y no una clasificación por programas. 
exposición de sus fines -lo que serían las metas y los grandes objetivos estratégicos en un sistema de presupuestación por programas- ${ }^{19}$.

En otros sí se rellena ese apartado, pero con una fórmula tan vaga y ambigua que realmente se refiere a las funciones y competencias que legalmente corresponden al centro administrativo, sin relación con el programa concreto que se expone a continuación $^{20}$.

En cuanto a los objetivos, brillan sorprendentemente por su ausencia en programas del tipo de aquellos con los que precisamente se creó y perfeccionó la técnica de la presupuestación por programas, como los de Obras Públicas -si bien es cierto que en los presupuestos de 2004 se ha mejorado notablemente y han comenzado a utilizarse de verdad las fichas de programas ${ }^{21}$.

${ }^{19}$ Vid pe programa 4131, Promoción y Protección de la Salud, Consejería de Salud, 2004.

${ }^{20}$ Pe de nuevo en el programa 6141/38, de Hacienda, se enumeran las funciones de la DG Tributos, servicio por servicio -así pe se nos informa de que al Servicio de Gestión Tributaria le corresponden la gestión y liquidación de los tributos propios y los cedidos, la implantación del sistema de información tributaria, la elaboración de declaraciones de IRPF a través del programa PADRE y el mantenimiento de relaciones con la Administración del Estado-. Lo cual pone de manifiesto una errónea comprensión de lo que significa la elaboración de un programa presupuestario, que en absoluto debe recoger bajo el mismo paragüas todas las funciones, por variadas que estas sean, del conjunto del organigrama de una Dirección General.

En el programa 6131, de la Intervención General, se explica que "el programa de gasto tiene por finalidad el control interno de la actividad económico-financiera de la CAR y la dirección y gestión de la contabilidad de la misma" y que "La Intervención General es, en consecuencia, un órgano encargado de promover la mejora de la gestión y de proporcionar una información fiable, completa e independiente sobre aquella a sus responsables últimos".

En el programa 4432, de Medio Ambiente, al menos se dice algo más (incluímos entre paréntesis los añadidos en la versión del programa para el año 2004): "Descripción: protección y mejora del medio ambiente del territorio propio de la Comunidad Autónoma de La Rioja (fomentando el desarrollo sostenible de las actividades y uso de los recursos naturales de una forma equilibrada y racional, desde una perspectiva de integración medio ambiental). Fines: (adopción de medidas preventivas ambientales en la implantación de las actividades) mejora de la calidad ambiental mediante una adecuada gestión de los residuos y vertidos (promover el uso de recursos de forma integral, fomento del desarrollo sostenible en el territorio de La Rioja)".

${ }^{21}$ Así en el programa 5121 la única información que se transmite en los presupuestos de 2003 es que “(1)as obras propuestas... para el año 2003 se agrupan en dos apartados: las correspondientes a segundas anualidades de obras de ejecución y las que están previstas contratar a lo largo del próximo ejercicio. Y se nos explican algunas -no todas- en estos términos: "El acondicionamiento de los corredores de la Red Regional Básica siguientes:

1. L-123, tramo Turruncún a la LR-283.

2. LR-115, tramo Arnedillo a la LR-484.

3. LR-113, tramo LR-435 a la LR 323”.

Sin embargo, en los presupuestos de 2004, siguiendo ortodoxamente el esquema propio de un presupuesto por programas, se indican varios objetivos, de entre el que destacaríamos el que aparece en tercer lugar: "(f)acilitar accesibilidad entre Comarcas y solucionar los tráficos de las travesías de los municipios de la Comunidad”. Los demás mencionados parecen más bien actividades (cumplimentar la ejecución del II Plan de Carreteras, acondicionar según características técnicas del Plan Red de Carreteras, conseguir mayor eficacia y rentabilidad para las inversiones programadas para las infraestructuras de carreteras).

Y en el programa 5132 se explicaba en los presupuestos de 2003 : "Se incluyen las segundas anualidades para las obras de acondicionamiento de riberas ya contratadas en San Millán de Yécora, Calahorra, Tirgo..." y nada más.

Pero en los presupuestos de 2004 se enumeran varios objetivos: planificación y gestión de inversiones, estudios para mejorar los cauces y el entorno de los ríos en los tramos urbanos de La Rioja, colaboración con Ayuntamientos para la recuperación de los ríos en el entorno urbano de los municipios y colaboración con Medio Natural para mejorar el entorno de los ríos en la Comunidad. 
En otros se intenta al menos establecerlos, aunque con muy desigual fortuna. En la mayor parte de los casos son simples enumeraciones de actividades ${ }^{22}$. Y en otros, de funciones que competen al ente administrativo ${ }^{23}$.

Ciertamente algunos se acercan a lo que debe $\operatorname{ser}^{24}$ y otros lo logran plenamente -todos los que hemos visto nosotros se refieren a Salud, curiosamente ${ }^{25}$ -

Las actividades se mezclan en muchos casos, como hemos indicado, con los objetivos $^{26}$; pero en otros programas aparecen perfectamente sistematizadas y ordenadas

Bien, realmente los estudios son necesarios para empezar a actuar con cierta racionalidad; se trataría, en nuestra opinión, de un objetivo instrumental, al servicio de los otro-, y verdaderos objetivos operativos serían la recuperación de los ríos y su entorno, mientras que la colaboración con otras administraciones es una simple actividad, por supuesto deseable.

${ }^{22}$ En el programa 4432, de Medio Ambiente, se recogen como objetivos, por este orden: "1. Desarrollo del Plan Director de Residuos de la CAR, 2. Ejecución del Plan de Saneamiento y Depuración de la CAR, 3. Desarrollo del Plan de Abastecimiento de agua potable de La Rioja, 4. Adopción de medidas de control de la contaminación atmosférica y vigilancia de la calidad del aire y cambio climático, 5. Adopción de medidas de control del ruido, 6. Adopción de medidas preventivas en materia de medio ambiente...”. En nuestra opinión, esto no son más que actividades. El único objetivo es el que se incluye en $7^{\circ}$ lugar: fomento del desarrollo sostenible.

${ }^{23}$ Es el caso del programa 6131, de la Intervención, que fija como "objetivos" los siguientes: “1. Ejercicio de la función interventora en todas las fases del gasto y de los ingresos de la CAR, 2. Control financiero en todas sus modalidades, 3. Dirección y gestión de la contabilidad pública de la CAR..."

${ }^{24}$ Los objetivos del Servicio de Gestión Tributaria -el problema es que el programa aglutina a todas las unidades administrativas, sin orden por funciones o fines o objetivos-, en el programa 6141/38 son: "Mejora en la gestión y liquidación de los tributos propios y cedidos, mejora en la calidad del servicio al contribuyente agilizando y simplificando los procedimientos de gestión y liquidación, permanente actualización en la formación del personal dependiente del servicio". El programa ganaría mucho si se ordenaran estos objetivos, distinguiendo entre operativos e instrumentales, y se jerarquizaran.

${ }^{25}$ El programa 4131 -promoción y protección de la salud-, el mejor de todos los analizados, en su versión de 2003, define hasta 11 objetivos y esta es, precisamente, la mayor objeción que podemos hacerle, puesto que según indican los expertos un programa no debe tener más de uno o dos objetivos (en la versión de 2004, la situación en mucho más confusa, puesto que se recogen nada más y nada menos que 44 objetivos, ordenados, además, por Subdirecciones Generales, lo cual es un error grave).

El primero (analizamos a continuación sólo la versión de 2003) es el siguiente: “... la mejora de la salud de la población riojana desde una perspectiva comunitaria, disminuyendo la carga de enfermedad -mortalidad, morbilidad y discapacidad- mediante la identificación de factores protectores y de riesgo, la acción directa y el trabajo con los agentes sociales. Todos ello para que, de una forma coordinada, se logre un efecto positivo sobre estos factores y el desarrollo de políticas saludables": lo cual muy bien podría ser la meta u objetivo estratégico, planteado, por cierto, a partir de una situación de necesidad detectada y delimitando -aunque sea someramente- la población afectada y beneficiaria del programa. Al mismo tipo pertenece el 3 "Fomentar la accesibilidad al sistema orientando los servicios sanitarios hacia el ciudadano, de forma que se logre el acceso efectivo a todas las prestaciones del sistema nacional de salud que son aceptadas conforme al estado del desarrollo tecnológico y el conocimiento científico; garantizando un diagnóstico preciso, pronóstico razonado y un tratamiento eficaz, cuando este sea posible, todo ello en un entorno de seguridad y confortabilidad".

Luego establece otros objetivos, algunos de los cuales, son objetivos operativos -“8. Elaborar planes sectoriales de acción que aseguren la equidad entre la población frente a cada situación contemplada en la intervención sectorial"; 9. "Establecer la coordinación necesaria entre equipos de atención primaria y farmacéuticos comunitarios de forma que las oficinas de farmacia integren acciones preventivas". Otros son objetivos instrumentales al servicio de la propia Administración -"5. Impulsar la formación continuada como instrumento de mejora de los procesos asistenciales y desarrollo de la organización sanitaria”-. Y otros, no son objetivos, sino meras actividades : "2. Proporcionar a todos los ciudadanos residentes en LR los identificadores sanitarios individuales", 10. "Verificar que en la realización de estudios clínicos se aplican los criterios establecidos que aseguran la calidad de los resultados y la protección a las personas incluidas en los estudios". 
por objetivos ${ }^{27}$-o por lo que el programa califica de objetivos que en muchos casos no lo $\operatorname{son}^{28}$-, aunque a veces se describen con un lenguaje telegráfico difícilmente comprensible por alguien ajeno al propio centro gestor $-\mathrm{y}$ no olvidemos que los parlamentarios autonómicos que han tenido que decidir sobre estos programas lo son- $\mathrm{y}$ mezclando actividades relevantes para la consecución de los objetivos con actividades puramente administrativas, que, aunque evidentemente imprescindibles, no deben aparecer en los presupuestos porque no aportan mayor información sobre la labor del ente en relación con el programa concreto que se tiene que valorar ${ }^{29}$.

Pero el elemento en el que más errores se comenten es, sin duda, en el de los indicadores. No es de extrañar, puesto que es muy difícil diseñar buenos indicadores que aporten información sobre las actividades, pero también sobre los resultados de los programas presupuestarios. Y lo es mucho más encontrar indicadores de impacto, que nos permitan valorar la medida en que el programa está solucionando o no la situación de necesidad, el problema económico-social que lo justifica.

Así el programa que mejor define sus indicadores -entre los que hemos estudiadoes, con diferencia, el 4131, Promoción y protección de la salud, que en su versión de 2003 y respecto a su objetivo $\mathrm{n}^{\circ} 1$, pe, incorpora indicadores como los siguientes: (1) gestión, adquisición y distribución de vacunas $\rightarrow \mathrm{n}^{\mathrm{o}}$ personas a vacunar cuantificado como porcentaje entre número de vacunados/población a vacunar o (2) difusión del programa de detección precoz del cáncer de mama: $n^{\circ}$ mujeres revisadas $/ n^{\circ}$ mujeres incluidas en el programa. Se trata de un programa que utiliza una gran cantidad de indicadores - la suma de la información obtenida es así muy relevante-, todos cuantificados, y en forma de porcentaje -no en forma de cifras absolutas, que casi nunca dicen nada-. La única crítica que cabría hacer es que no recogen una proyección plurianual de los mismos - probablemente no lo piden las instrucciones elaboradas por la Consejería de Hacienda-.

${ }^{26}$ Es lo que sucede en los programas 5121 y siguientes, de Obras Públicas en el presupuesto de 2003 mucho más que en el de 2004 (aunque en este año el error es tan patente a veces como en el programa 5131, Gestión de Infraestructuras Hidraúlicas, que la gestión de las presas competencia de la CA se cita tanto como objetivo $-\mathrm{n}^{\circ} 5-$, como más adelante como actividad $-\mathrm{n}^{\circ} 6-$.

${ }^{27}$ Es un estupendo ejemplo el programa 4131, de Salud, del año 2003, que menciona en relación con el primer objetivo (mejora de la salud de la población riojana...), pe, actividades como "mantener el Sistema Regional de Vigilancia Epidemiológica", "mantener el Sistema de Alerta Sanitaria” o "Seguimiento y control del programa de Salud Bucodental”.

${ }^{28}$ Como sucede en el programa 6131 de la Intervención, que menciona la "fiscalización previa y limitada de todos los actos administrativos que impliquen la realización de un gasto", "la intervención formal y material del pago", la "elaboración de los informes preceptivos a la elaboración de normas con contenido económico".

Vid también el programa 4432 del año 2003 , de Medio Ambiente, en relación con su $6^{\circ}$ objetivo, actividades como "desarrollo de campañas de concienciación como apoyo a la gestión de residuos y depuración de aguas residuales; organización de la información sobre Medio Ambiente LR, edición de publicaciones y material divulgativo...”. O en ese mismo programa para 2004, el objetivo 1 -desarrollo del Plan Director de Residuos de la CA-, con actividades como sellado y clausura de vertederos, concurso en la gestión de residuos agropecuarios, actuaciones en relación con suelos contaminados, control de residuos industriales...

${ }^{29}$ Es lo que sucede en el programa $6141 / 38$, de Hacienda, donde se recogen como actividades desde la "reforma plurianual del sistema de ingresos e implantación progresiva del mismo a través del Proyecto Oria" hasta la "(i)mplantación del código de barras" o la "normalización de procedimientos y documentos", pasando por "programación y desarrollo de un plan de formación específica tributaria", pero también la "edición de folletos informativos acerca de las deducciones autonómicas relativas al IRPF...". 
En otros programas los indicadores no son tan buenos ${ }^{30}$, no proporcionan información relevante ${ }^{31}$, no aparecen cuantificados - esto es, no fijan el criterio de éxito con el que comparar la gestión realmente efectuada en el ejercicio presupuestario por el ente, para poder valorarla- ${ }^{32}$, etc. Pero lo más sorprendente y criticable es que numerosos programas ni siquiera los contienen ${ }^{33}$.

\section{EFECTOS DE LA APROBACIÓN DE LA NUEVA LEY GENERAL PRESUPUESTARIA DEL ESTADO EN LA RIOJA}

Si la CA no lo evita -mediante una Ley de Hacienda Pública propia o utilizando cualquier otra ley, como indicábamos en la introducción-, la nueva LGP se aplicará en

${ }^{30}$ Véanse los del programa 3111 para 2003, Administración General de Servicios Sociales (que en realidad parece agrupar varios, 3111, 3121, 3122 y 3123), que incluye como indicadores la"negociación de convenios con corporaciones locales antes del 31-3-2003", la "construcción y equipamiento Centro SS (Rioja Baja) antes del 31-12-2003" o la "realización jornada del voluntariado antes del 31-10-2003", entre otros. En la versión de 2004 cita como indicadores "número de reuniones y Comisiones de Dirección efectuadas", "número de reuniones efectuadas, elaboración de instrucciones y circulares, etc" -sic- o "fijación de protocolos comunes, seminarios, reuniones, jornadas", ninguno de los cuales aporta la más mínima información.

31 Programa 6141/38, de Hacienda, Servicio de Inspección Tributaria y Valoración: número de inspecciones realizadas, $\mathrm{n}^{\mathrm{o}}$ de actas de conformidad, $\mathrm{n}^{\mathrm{o}}$ de actas de disconformidad, $\mathrm{n}^{\circ}$ expedientes sancionadores, $\mathrm{n}^{\mathbf{0}}$ expedientes de inspección... Todos ellos indicadores de actividad -en cifras absolutas, que no proporcionan mucha información-, pero no de resultados, ni de impacto. Además no aparecen cuantificados, esto es, no se fija ningún "criterio de éxito", no se establece el nivel de eficacia o eficiencia al que se pretende llegar, de modo que no podrá nunca valorarse la ejecución real del programa durante un año determinado, ni podrá calificarse de adecuada o imperfecta.

${ }^{32}$ Vid programa 6141/38, de Hacienda, que en relación con el Servicio de Gestión Tributaria, recoge los siguientes $-\mathrm{y}$ así de indefinidos-: número de autorizaciones, número de contribuyentes atendidos, volumen de deuda tributaria, número de expedientes, número de notificaciones...

O el programa 4111, Administración General de Salud, con algunos indicadores como número de reuniones, elaboración de instrucciones, número de reglamentos tramitados en desarrollo de la Ley de Salud, número de informes... (en algún caso sí se recogen indicadores útiles como el "resolución de, al menos, el $85 \%$ de expedientes y procedimientos en los plazos fijados", el 3.2.1, en relación con el objetivo 3: "agilización administrativa en general y, en especial, en los expedientes relacionados con usuarios y terceros atendiendo a los principios de eficacia, eficiencia y servicio a los ciudadanos".

${ }^{33}$ Pe programa 4432, de Medio Ambiente (el de 2004 sí contiene indicadores, aunque no estén ordenados por objetivos -siendo estos muy diferentes entre sí y sin que quede claro si todos los indicadores operan respecto a todos los objetivos y cómo se distribuyen, pues hay 7 objetivos y 14 indicadores,y algunos sean tan ininteligibles como "población con EDAR" -estaciones depuradoras de aguas residuales, suponemos-, "MI de colector construido" o "N" fallos/semana toma datos Red de Control de Calidad del Aire"; otros tan poco útiles para valorar la actuación de la Administración como "tn de residuos" o "m3 de agua tratada" -puesto que las cantidades de residuos que se generen en la Comunidad o los metros cúbicos de agua que se utilicen dependen de un conjunto de variables incontrolables para la Administración, que nada tienen que ver con su comportomiento eficaz y eficiente- y en general todos poco significativos).

O todos los programas de Obras Públicas (en 2004 sí aparecen indicadores pero tales como volumen de inversión, número de expedientes tramitados, núcleos con abastecimiento resuelto y número de presas en explotación en el programa 5131 de Gestión de infraestructura hidraúlica, y número de kilométros en que se actúa, volumen de inversión, tipos de actuaciones y poblaciones afectadas en el 5121, Creación de Infraestructura de Carreteras, o número de expedientes tramitados, volumen de inversión ejecutada y número de poblaciones en las que se ha actuado en el 4311, Promoción y ayuda para la construcción, rehabilitación y acceso a la vivienda).

O el mismísimo programa 6131, de Control Interno, de la Intervención, ni en el programa de 2003, ni en el del 2004 - ¿si todos los centros gestores siguen su ejemplo, cómo va a desarrollar su función de control financiero?. 
La Rioja como Derecho supletorio, y obligará a utilizar una nueva técnica presupuestaria, la gestión por objetivos, en el conjunto de la Administración autonómica ya en 2005. Y esto no podrá evitarse con simples órdenes y resoluciones de la Consejería de Hacienda dando instrucciones a los centros gestores para que continúen preparando presupuestos por programas. De modo que conviene reflexionar sobre lo que implican las innovaciones que introduce la nueva Ley.

¿En qué consiste realmente la nueva técnica presupuestaria? Si analizamos los preceptos de la nueva Ley General Presupuestaria donde se hace referencia a ella, observaremos que:

En primer lugar, se quiere situar la gestión anual de recursos públicos en el marco de una planificación plurianual a tres años -aunque anualmente actualizada-. Por ello se pide a los Departamentos Ministeriales - a las Consejerías de la CA- que elaboren unos "programas plurianuales" -art. 29.1-, en base a los cuales se prepararán los escenarios presupuestarios plurianuales, en los que se intentará determinar la evolución de las grandes variables económicas, para poder establecer previsiones sobre la evolución de ingresos públicos y decidir los recursos que podrán asignarse a las distintas políticas de gasto, esto es, a los grandes fines de gasto público, en función de las prioridades decididas por el Gobierno. Los programas plurianuales del Departamento Ministerial -o Consejería- serán desagregados en programas, también plurianuales, de cada uno de los centros gestores dependientes del Ministerio -art. 35.2-.

Sin embargo, el presupuesto seguirá siendo anual -como no podía ser menos dado lo dispuesto en el art. 134 CE y mientras no se reforme la Constitución-. Anualmente, pues, se elaborará un presupuesto incardinado en el seno de los escenarios presupuestarios, en el que se fijarán los "objetivos" de los centros gestores y los recursos que se les asignan para ellos. Los programas plurianuales se desarrollarán, por tanto, o concretarán en "programas anuales" que serán el conjunto de recursos puestos a disposición del ente para la consecución de un objetivo -art. 35.4-. Se mantiene, pues, la clasificación por programas, junto con la orgánica y la económica -art. 39-.

En segundo lugar, se definen los elementos que integran un programa. Así pe se nos informa de que los "programas plurianuales" -art. 29.6 y 7- deben definir los objetivos del ente gestor -definidos de forma objetiva, clara, mensurable-; las actividades precisas para alcanzar los objetivos; los medios -económicos, materiales y personales-; las inversiones -reales y financieras-, y los indicadores -para la medición, seguimiento y evaluación de los objetivos desde el punto de vista de la eficacia, eficiencia, economía y calidad de la actuación pública-. Y deben estar coordinados con los planes sectoriales u otro tipo de programas de actuación vigentes en cada Ministerio -o Consejería-.

Más adelante -art. 35.2- se añade que los programas plurianuales recogerán el gasto autorizado para la consecución -mediante el desarrollo de las actividades oportunas- de objetivos que pueden ser de distinto tipo: de producción de bienes o servicios públicos, de cumplimiento de obligaciones específicas o de realización de otras actividades. Y hablan también de objetivos otros preceptos que distinguen entre "objetivos estratégicos" -art. 28.1 y 4, 29.1, 70.1-, "objetivos finales" -art. 70.2- y "objetivos instrumentales" -se señala expresamente que estos últimos deben ponerse en 
relación con los primeros, puesto que se trata de objetivos secundarios que sirven sólo para alcanzar los principales, en el art. 31-.

En tercer lugar, se regula un tipo especial de programa, el programa de apoyo, para las actividades de los servicios horizontales, que se encargan de colaborar con el resto de los órganos administrativos, desarrollando una labor instrumental respecto a estos -art. 35.3-.

En cuarto lugar, se advierte en reiteradas ocasiones que los recursos en presupuestos sucesivos se asignarán en función de los resultados conseguidos en años anteriores -art. 35.4, 69-. Es evidente que para ello se necesitará contar con buena información, no sólo cuantitativa, de modo que no sólo el presupuesto inicial, sino también sus modificaciones deben permitir conocer en todo momento si se están cumpliendo o no los objetivos fijados, detectar desviaciones, conocer sus causas -vid art. 27.5, 35.4, 47.3, 69.1, 70.1-.

En quinto lugar, se establece claramente la responsabilidad de los titulares de los entes públicos/órganos administrativos respecto a la consecución de los objetivos fijados -art. 69.4- en el marco de un sistema de gestión orientado a los resultados -art. 69.1 y 2-. Por eso deben prepararse cada año, al liquidar los presupuestos un balance de resultados y un informe de gestión, complementados con un memoria, con información sobre los resultados conseguidos en cuanto a los objetivos previstos -art. 71.1, $128.4 \mathrm{y}$ 5-, que se incorporarán a la Cuenta General del Estado -o de la CA; art. 130.2-.

En sexto lugar, se prevén distintos tipos de controles y evaluaciones. La evaluación -art. 72- debe valorar la consecución por los entes públicos de sus objetivos estratégicos y el impacto socioeconómico. Los controles serán desarrollados en parte por los propios centros gestores -sistema de seguimiento de objetivos, que no se regula pero es mencionado en el art. 125 o en el 126, en el que se deberán respetar los principios y criterios establecidos por la IGAE- y, en parte por la Intervención General de la Administración del Estado -o la Intervención de la CA-, cuyas potestades y técnicas son ampliamente reguladas en la nueva Ley, incorporando la regulación hasta ahora contenida en reglamentos ${ }^{34}$.

De entre las posibilidades de actuación de la Intervención destacaríamos el control financiero -159.1 , con informes de actuación en los que pueden proponerse medidas correctoras, de cuyo seguimiento también ha de encargarse la Intervención, art. 161.3-, y las auditorías operativas -art. 164.1.c y 170-, que incluyen auditorías de programas -art. 170.1-, auditorías de sistemas y procedimientos -art. 170.2- y auditorías de economía, eficacia y eficiencia -art. 170.3-.

En séptimo y último lugar, se presta mayor atención a la difusión de los resultados del control y, por tanto, de los resultados de la gestión pública en relación con los objetivos. En la nueva Ley no sólo se entregará información mensualmente a las Cortes -art. 135, o al Parlamento autonómico-, sino que se publicará en el BOE -o el Boletín Oficial de la CA- y hasta, si la IGAE lo considera oportuno, en otros medios -art. 136-.

34 RD 2188/1995, 28-diciembre, sobre el régimen de control interno ejercido por la Intervención General de la Administración del Estado, básicamente. 
Esta regulación legal ya es mucho más detallada que la que se contenía en relación con la estructura y procedimientos de elaboración de los presupuestos básicamente en los art. 52-54 del TR LGP de 1988, normas tan vagas que han permitido de hecho la utilización de varias técnicas, aplicadas de forma sucesiva y simultánea en el tiempo, técnicas como el presupuesto por tareas, el presupuesto por programas y el presupuesto en base cero limitado ${ }^{35}$.

Aun siendo la regulación contenida en la ley más completa, no está totalmente cerrada y deberán ser los reglamentos quienes se encarguen de cuestiones como la regulación del procedimiento de elaboración y la estructura de los programas plurianuales de los Ministerios -art. 29.5, o Consejerías- y de los Centros Gestores -art. 30.2-; el procedimiento de elaboración de los presupuestos -art. 36.2-; la composición de la Comisión de Políticas de Gasto -art. 36.2.primera ${ }^{36}$; o el contenido y estructura de los documentos que deben formar parte de las cuentas anuales de los entes públicos -art. 128.6-. Y esto para La Rioja significa que la Comunidad Autonóma podrá intervenir normativamente -mediante órdenes de la Consejería de Hacienda, pe-, pero no para desmarcarse del nuevo sistema, sino para intentar adaptarlo a las necesidades y peculiaridades de su administración. La cuestión es ¿está preparada para hacerlo?

Para empezar a nosotros, la nueva regulación presupuestaria nos parece un tanto confusa y no del todo realista y bien encaminada.

Confusa porque si lo que se quiere es establecer un sistema de gestión y presupuestación por objetivos -art. 70 nueva LGP-, no tiene sentido, a nuestro juicio, que se siga incluyendo la clasificación presupuestaria por programas -vinculante, como las otras 37 -, puesto que en un modelo como el que se pretende establecer el gestor se responsabiliza de la consecución de unos determinados resultados tomando como referencia unos objetivos previamente fijados y eso es todo. La asignación concreta de los recursos recibidos es decidida libremente por él -responde después por ello-. De ahí los grandes márgenes de autonomía en la gestión y la flexibilidad que tanto se valoran

${ }^{35}$ De nuevo tenemos que remitirnos por razones de espacio a nuestros trabajos, "El presupuesto por programas..." y "Hacia una gestión por objetivos...".

${ }^{36}$ Por cierto que no se mencionan ya las Comisiones de Análisis de Programas, ni el Comité de Ingresos Públicos.

37 Aunque esa vinculación seguirá siendo problemática, como lo es ahora. En efecto, ya hemos comentado que las memorias de objetivos y programas de los Departamentos Ministeriales en el Estado y de las Consejerías en la CA no se publican junto con el texto de la ley de presupuestos generales del Estado cada año y que, en nuestra opinión, eso priva a los programas de fuerza jurídica, puesto que en la ley sólo aparece un anexo con el nombre -normalmente bastante ambigüo, por cierto- y nada más, de cada programa, y los recursos asignados según la clasificación económica.

Pues bien, la nueva LGP, en el art. 37, se dice que el proyecto de Ley de Presupuestos anual enviado a las Cortes -al Parlamento autonómico- constará de una parte articulada y de unos anexos, entre los cuales se incluirá la clasificación por programas. Pero la descripción detallada de los mismos -su contenido, objetivos, actividades, indicadores, etc- sólo aparecerá en una memoria descriptiva que es calificada de simple "documentación complementaria", de modo similar a como hace el actual art. 54.tercera.b TR LGP, aunque este artículo es más vago y permite exigir, como hacemos nosotros, que se lleve a la ley la descripción completa de los programas, para dotarles de eficacia jurídica.

El art. 37 se remite a los art. 40 -estructura de los estados de gastos de los PGE- y 41 -estructura de los estados de ingresos-. El art. 40 se remite, a su vez al 35 -los créditos y los programas presupuestarios- y exige, al hablar de la clasificación por programas, que los créditos para gastos se agrupen en función de los objetivos plurianuales y anuales del centro gestor. Así que al menos información sobre los objetivos sí recibirá el Parlamento y sobre ellos decidirá con valor legal, pero ¿sobre algo más? 
como ventajas de este sistema. Esto es, un presupuesto por objetivos es el que resultaría de lo dispuesto en el art. 35.4 de la nueva LGP; no se derivaría tan claramente de lo dispuesto en el art. 29.6. Y es que, parece, el legislador sigue aún muy apegado al esquema del PPBS. O a alguna de sus categorías, como la de "programa" o, al menos, a la denominación a la que no quiere renunciar, pese a que ello supone introducir ciertas dosis de confusión.

En nuestra opinión, lo mejor hubiera sido -sería- mantener varias técnicas presupuestarias vigentes simultáneamente - de ahí que no nos parezca demasiado bien encaminada la reforma, por unidireccional y generalista-. Por ejemplo, como se advierte en el art. 35.3 de la nueva LGP, es claro que las actividades horizontales, de apoyo al resto de los órganos administrativos, que desarrollan las Secretarías Generales Técnicas de los Ministerios no pueden someterse al mismo esquema de funcionamiento que siguen los centros gestores encargados de la provisión de bienes y servicios tangibles, directamente accesibles a los ciudadanos. La nueva LGP parece renunciar en este ámbito al sistema de gestión por objetivos y prevé que se utilicen unos "programas de apoyo" especiales -no explica demasiado en qué consistirían-. Nosotros pensamos que ni siquiera esto es adecuado, porque la realidad ha demostrado que precisamente estas actividades no pueden ordenarse en forma de programas y que respecto a ellas lo mejor sería emplear un presupuesto por tareas -que es de hecho lo que sucede aunque no quiera reconocerse-.

Y en otras áreas podría pensarse en mantener el actual presupuesto por programas, muchos de cuyos elementos se recogen en la nueva ley -planificación plurianual, selección de objetivos estratégicos y operativos, ordenación de actividades, indicadores, seguimiento y control como claúsula de cierre del sistema-. Porque el presupuesto por programas, el presupuesto por tareas y la gestión por objetivos son técnicas perfectamente compatibles y complementarias, que responden a los principios del art. $31 \mathrm{CE}$. Aunque ninguna de ellas es perfecta, todas ofrecen ventajas en ciertas áreas. En cuáles opera mejor cada una es lo que habría que ir pensando, a nivel estal y autonómico.

Por último nos parece que la reforma legislativa puede pecar, una vez más, de poco realista. $\mathrm{Y}$ es que el legislador español no parece haber aprendido aún esta importante lección: que no se cambia una Administración de la noche a la mañana mediante la simple promulgación de una ley ${ }^{38}$. Probablemente este no es problema sólo del legislador español, pero es suyo también. Y resulta imperdonable a estas alturas, cuando después de veinte años de reforma presupuestaria es evidente para todos que un cambio de rumbo sólo es posible si se plantea a largo plazo - se trata de cambiar comportamientos de una pluralidad de agentes con intereses en conflicto e interrelacionados de forma compleja- y se introduce progresivamente -como se hizo acertadamente en el Estado a partir de 1984 con el PPBS-.

De modo que no tiene ningún sentido que se pretenda acelerar la tramitación y entrada en vigor de la nueva LGP para que los presupuestos de toda la Administración del Estado -y de la Comunidad Autónoma de La Rioja- de 2005 sean ya preparados de acuerdo con el nuevo sistema de gestión por objetivos. Es preciso suministrar los

\footnotetext{
${ }^{38} \mathrm{Y}$ además es un problema general como nos demostró hace tiempo A. NIETO en su trabajo "La nueva organización del desgobierno", Ariel, 1996.
} 
conocimientos y el apoyo necesarios -logístico y de todo tipo- a los agentes participantes, es importante conseguir que se involucren y acepten hacerlo activamente, que sientan como suyo el nuevo sistema, que entiendan su utilidad para ellos mismos como gestores responsables de intereses públicos, y aportarles liderazgo y modelos. Es necesario contar con los instrumentos precisos, como una buena contabilidad analítica y de costes de la que, a pesar de todos los avances logrados en los últimos años, aún no disponemos. Es imprescindible que la Administración aprenda a planificar su gestión a medio plazo y no se deje anular por la presión del día a día, que delimite mucho mejor sus objetivos -elemento ya importante en el sistema de presupuestación por programas, y absolutamente fundamental, por único, en un presupuesto por objetivos- y que con ayuda de expertos configure buenos indicadores de gestión, no sólo cuantitativos y de proceso -que son los que ahora proliferan, a pesar de su más bien escasa utilidad-, sino cualitativos y de resultados, y más aún de impacto, porque sin ellos será imposible comprobar cuáles han sido los efectos de la acción pública para exigir, en función de ellos, responsabilidad a los gestores.

En definitiva, en nuestra opinión, ciertamente sería deseable mejorar e incluso reformar muy a fondo el modo en que parte de la Administración estatal y, más aún, autonómica española está presupuestando y gestionando recursos públicos. Pero no creemos que las actuales deficiencias se deban a que la técnica que según la normas se emplea, el presupuesto por programas, sea inadecuada -no lo es en la mayor parte de los casos-, sino a que sencillamente esta técnica no se está aplicando. O se está aplicando de forma sumamente incorrecta. Sería preciso comprobar en qué supuestos el PPBS no es la técnica adecuada y en cuáles sí, en qué casos pueden mantenerse los programas actualmente existentes y en cuáles deben mejorarse la definición de objetivos, la ordenación de actividades, los indicadores, etc. Analizar en que otros supuestos no puede emplearse un sistema de presupuestación por programas, porque no resulta conveniente dada la naturaleza de las funciones o el tipo de órgano administrativo o las circunstancias en que debe actuar y pensar entonces por qué otra técnica debiera sustituirse, si por presupuesto por tareas o por un sistema de gestión por objetivos. Y debería pensarse en cómo introducir y aplicar estas formas de gestión -sobre todo en el segundo caso, por novedosa- y con qué mecanismos de control deberían acompañarse para garantizar en todo momento que la defensa de los intereses públicos queda a salvo.

Lo verdaderamente relevante en todos los casos es que tanto los gestores, como los responsables políticos -en el gobierno y en el Parlamento- y los ciudadanos sepamos en todo momento por qué y para qué se están gastando unos recursos que nos pertenecen a todos, esto es, que se sepa cuál es la situación de necesidad que justifica la intervención pública, cuáles son los objetivos perseguidos y cuáles están siendo los resultados, en términos de bienes y servicios públicos -outputs- y de impacto o resolución de la situación de necesidad inicial -outcome-. Y que esta información, en la medida de lo posible, se vaya obteniendo a medida que la Administración actúa, para que sea posible detectar las desviaciones y corregirlas cuando aún se está a tiempo, o comprobar que el problema ya ha desaparecido y la acción pública no es necesaria - o lo es en menor medida- o que la actuación de la Administración no está resolviendo el problema en absoluto, para que sea posible su completo replanteamiento. 
Sólo procediendo de esta manera se respetarán los principios de economía, eficacia y eficiencia recogidos en nuestra Constitución y ahora también muy citados en la nueva Ley General Presupuestaria.

\section{CONCLUSIONES: ¿ES DESEABLE AVANZAR HACIA UNA GESTIÓN POR OBJETIVOS?}

A lo largo de este artículo hemos intentado demostrar que las técnicas empleadas en la elaboración de los presupuestos públicos cada año no son de importancia secundaria. La forma en que se presenta la información en el proyecto de Ley de Presupuestos Generales de la Comunidad Autónoma, relativa a las actividades que todos y cada uno de los centros gestores de la Administración Pública pretenden desarrollar durante el año siguiente, condiciona el conocimiento y asimilación de la información que el Parlamento puede llevar a cabo, y todo el proceso de discusión de la ley más importante aprobada cada año.

Esa ley se convierte en el marco en el que debe desarrollarse la gestión pública y es el punto de referencia del control del gasto público, interno o externo, simultáneo a la gestión o posterior. Y los resultados de este control son parte de la información que se empleará en la elaboración de los presupuestos de años sucesivos. Así pues, la técnica de presupuestación influye no sólo en la elaboración y aprobación de los presupuestos, sino en todo el proceso de gestión del gasto público. Y debe responder a los valores de equidad, por un lado, economía, eficacia y eficiencia, por otro -art. 31.2 CE-.

Hasta ahora nuestra Administración ha venido utilizando el sistema de presupuestación por programas en teoría, aunque, como hemos tenido oportunidad de comprobar, en muchos casos se emplea un presupuesto por tareas, aunque no se reconoce así expresamente, y en otros muchos los programas no respetan la estructura que según los teóricos y las órdenes anuales de la Consejería de Hacienda con las instrucciones para la elaboración de los presupuestos, deberían tener. No se distingue claramente entre objetivos y actividades, no se fija un objetivo estratégico al servicio del cual se articulen y ordenen objetivos operativos, todos debidamente definidos y jerarquizados y, sobre todo, no se fijan buenos indicadores con los que sea posible comprobar el grado de eficacia del programa, esto es, si se están cumpliendo los objetivos o no y, en este caso, por qué es así. Además hemos podido observar que la mayoría de los programas se mantienen sin cambios año tras año, lo que nos hace sospechar que ni los gestores, ni los responsables políticos -en el gobierno y en el Parlamento- se preguntan realmente por la razón de ser de los programas existentes, dándola por supuesta como en un presupuesto tradicional incrementalista de medios.

La nueva Ley General Presupuestaria aprobada en noviembre de 2003 pretende dar un nuevo impulso al proceso de modernización de nuestra Administración y aumentar -se dice en su exposición de motivos- la racionalización y economía, eficacia y eficiencia de nuestro sistema de gestión del gasto público. Y opta para ello por reformar la técnica presupuestaria, sustituyendo la presupuestación por programas -aunque el nombre de esta categoría al menos se mantiene- por un sistema de gestión por objetivos. 
Con él se pretende fomentar la responsabilidad de los gestores por los resultados de su actuación y a cambio se les ofrece mayor autonomía y flexibilidad.

Nosotros dudamos de que este cambio vaya a mejorar realmente los procesos de gestión de recursos públicos en nuestro país. Ni este sistema es el óptimo en todos los casos, puesto que la Administración está compuesta por un conjunto de órganos y centros de muy distinta naturaleza y estructura, encargados de desarrollar funciones diferentes, que necesitan formas de actuación y presupuestación distintas adaptadas a sus necesidades. Ni nuestra Administración está preparada para su implantación inmediata y simultánea en el conjunto de la misma -ya para los presupuestos de 2005 como se pretende-, porque si la aplicación real que está teniendo lugar del PPBS demuestra que muchos de los centros gestores no saben definir bien sus objetivos de actuación y presupuestarios, y por ello aplican de forma incorrecta el sistema de presupuestación por programas, difícilmente mejorarán con una técnica nueva que desconocen -no se les está dando tiempo para adaptarse a ella-.

Es más, la amplia autonomía que quiere reconocérseles puede llegar a resultar peligrosa para los intereses públicos -y no queremos pecar de alarmistas-, si el contrapeso que supone la evaluación y el control de su gestión por los resultados depende de una -en nuestra opinión- hoy por hoy imposible buena definición de objetivos en el conjunto de la Administración, por un lado, y de una correcta medición y valoración de resultados también actualmente de muy difícil práctica, por otro.

Mejor hubiera sido, en definitiva, haber intentado primero solucionar los problemas que existen en la aplicación del PPBS, sobre todo en la definición de objetivos e indicadores -para medir los resultados-, y luego pensar en la introducción progresiva del MBO sólo en aquellos ámbitos - ¿acaso ha de emplearse una única técnica presupuestaria en el conjunto de la Administración?- en los que pueda ser útil y adecuada para dar cumplimiento a los principios del art. 31.2 CE.

La Administración del Estado no tendrá más que remedio que aplicar la nueva ley ya en vigor en lo que respecto a los preceptos que regulan la nueva técnica presupuestaria, pero la de la Comunidad Autónoma de La Rioja aún tiene una oportunidad para evitarlo. El legislador autonómico debería aprovechar este momento para dictar esa Ley propia de Hacienda Pública que echamos de menos para regular, de la manera más adecuada al contexto y características de nuestro territorio y nuestra Administración, los procesos de elaboración de los presupuestos, gestión y control del gasto público, con una combinación flexible de técnicas - presupuesto por tareas y por programas básicamente- que permitan a los distintos órganos administrativos gestionar los recursos públicos con equidad y de forma económica, eficiente y eficaz -art. $31 \mathrm{CE}-$, pero al mismo tiempo realista y ajustada al principio de legalidad -103 CE-. 